\title{
Carpet Wear Classification based on Support Vector Machine Pattern Recognition Approach
}

${ }_{1.2}$ C. Copot, ${ }_{2}$ S. Syafiie, ${ }_{3}$ S. Vargas, ${ }_{2}$ R. de Keyser, ${ }_{4} \mathrm{~L}$. van Langenhove, ${ }_{1} \mathrm{C}$. Lazar

1Department of Automatic Control and Applied Informatics, "Gheorghe Asachi" Tehnical University of lasi

2 Department of Electrical energy, Systems and Automation, Ghent University

${ }_{3}$ Department of Telecommunication, Ghent University

${ }_{4}$ Department of Textile, Ghent University 


\section{Outline}

- Introduction

- Features extraction

- PCA (Principal Component Analysis)

- Features classification

- Results

- Conclusion 


\section{Objective}

\section{Introduction}

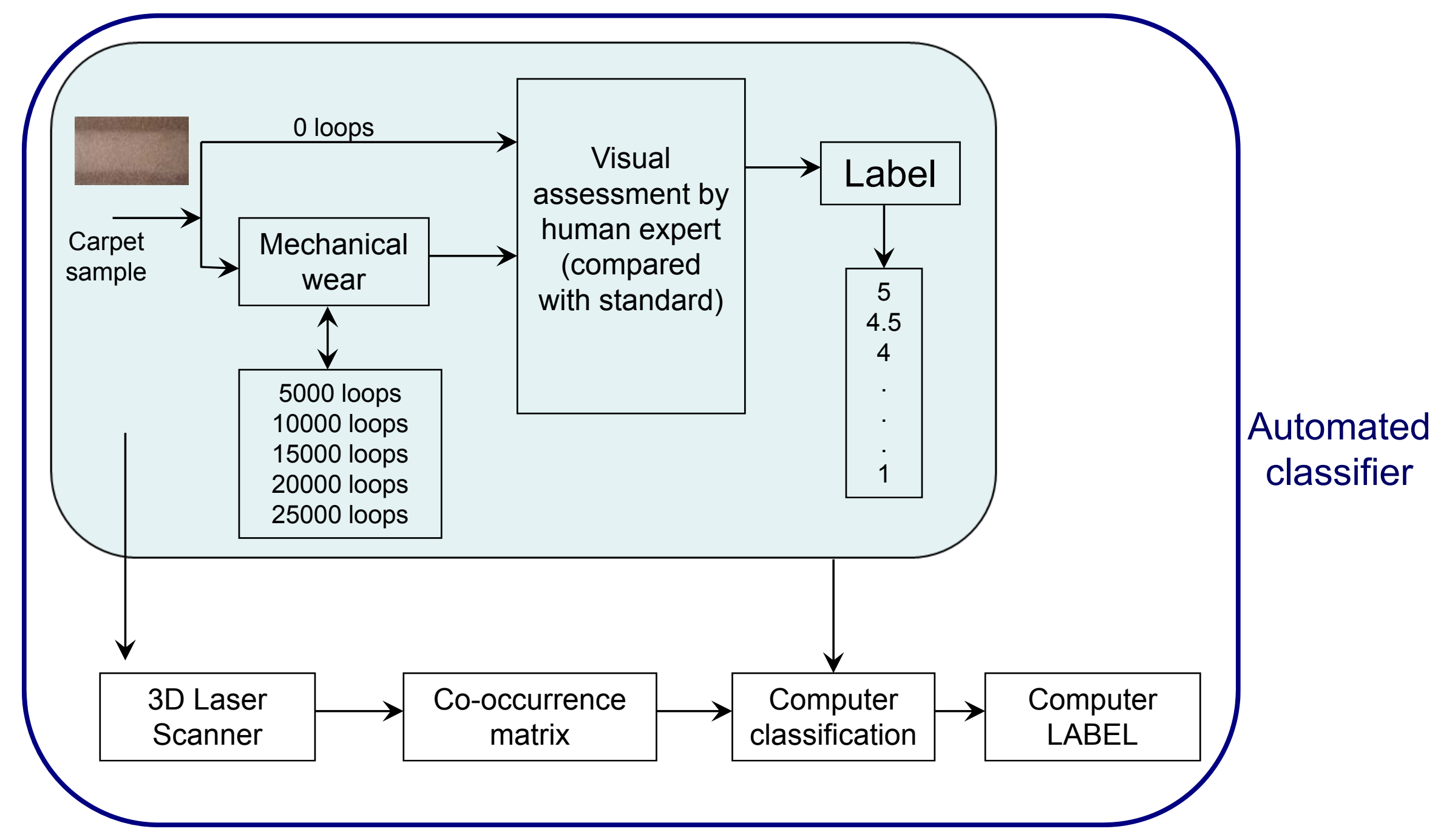

Cosmin COPOȚ - "Gheorghe Asachi" Tehnical University of Iasi 


\section{Introduction}

\section{Data Acquisition}

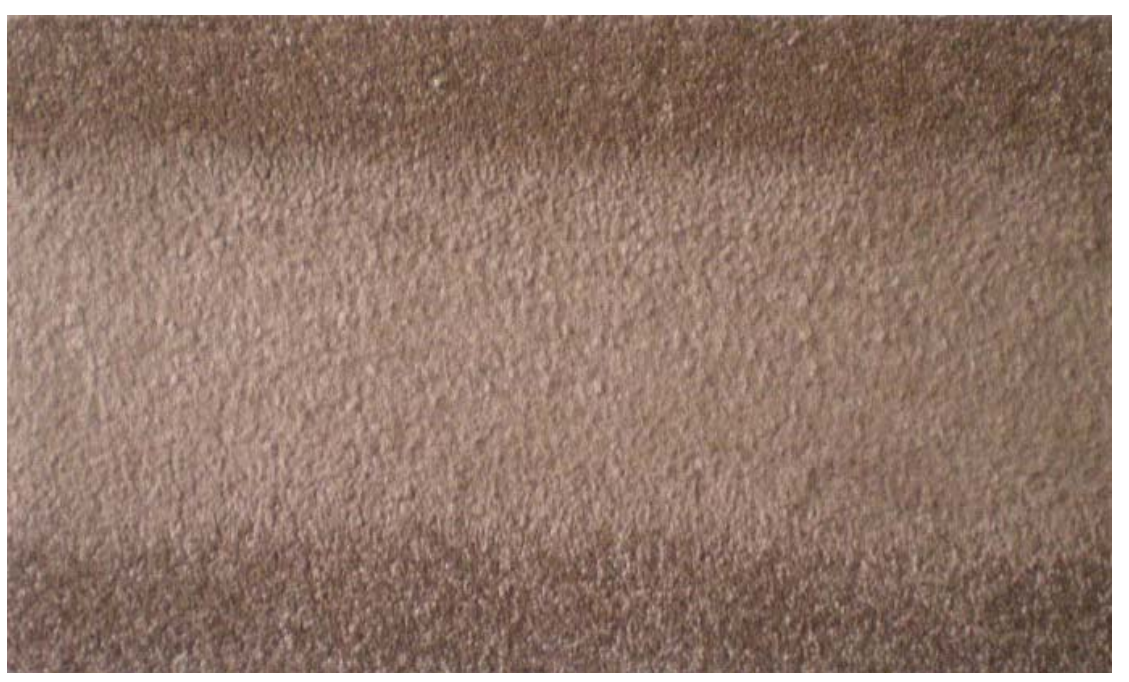

Fig 1. Digital image

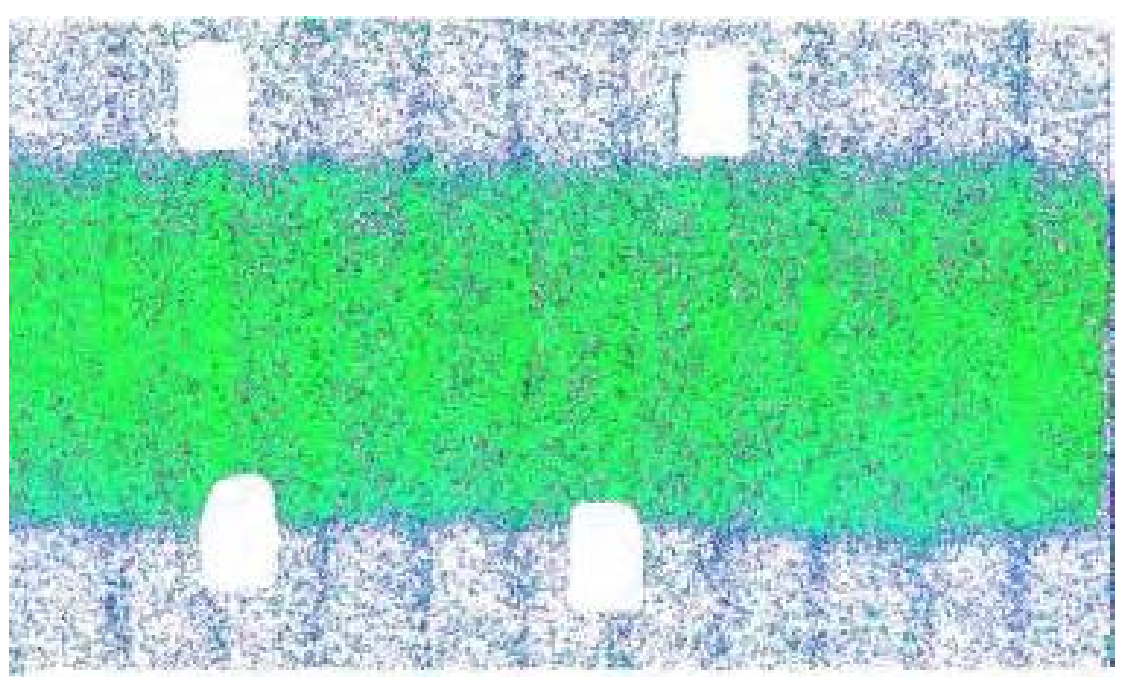

Fig 2. Laser image 


\section{Co-occurrence matrix}

$>$ Co-occurrence matrix composed

\begin{tabular}{|c|c|c|c|c|c|c|c|c|c|c|c|c|}
\hline & & & & & & & 2 & & 4 & 5 & 6 & \\
\hline & & & & & 1 & 2 & 3 & 1 & 0 & 0 & 0 & 0 \\
\hline 1 & 3 & 4 & 2 & 7 & & 0 & 0 & 0 & 0 & 1 & 0 & 2 \\
\hline 1 & 1) & 2 & 5 & 6 & 3 & 0 & 0 & 0 & 1 & 0 & 1 & 0 \\
\hline & & & & & 4 & 0 & 1 & 0 & 0 & 0 & 0 & 0 \\
\hline 3 & 6 & 2 & & 2 & 5 & 0 & 0 & 0 & 0 & 0 & 1 & 0 \\
\hline 1 & 1 & 2 & 7 & 5 & 6 & 0 & 0 & 0 & 0 & 0 & 0 & 1 \\
\hline & & & & & 7 & 1 & 0 & 0 & 0 & 1 & 0 & 0 \\
\hline
\end{tabular}

Co-occurrence matrix $\left(0^{\circ} \& d=1\right)$

$C_{d}(g(i, j), g(i+k, j+l))=C_{d}(g(i, j), g(i+k, j+l))+1$

Where: $\quad d=\sqrt{k^{2}+l^{2}}$ 


\section{Haralick's Features}

$\checkmark$ Energy

$\checkmark$ Sum Entropy

$\checkmark$ Entropy

$\checkmark$ Sum Variance

$\checkmark$ Inertia

$\checkmark$ Difference Variance

$\checkmark$ Homogeneity

$\checkmark$ Difference Entropy

$\checkmark$ Contrast

$\checkmark$ Informational Measures of Correlation

$\checkmark$ Correlation

$\checkmark$ Maximal Correlation Coefficient

$\checkmark$ Sum Average

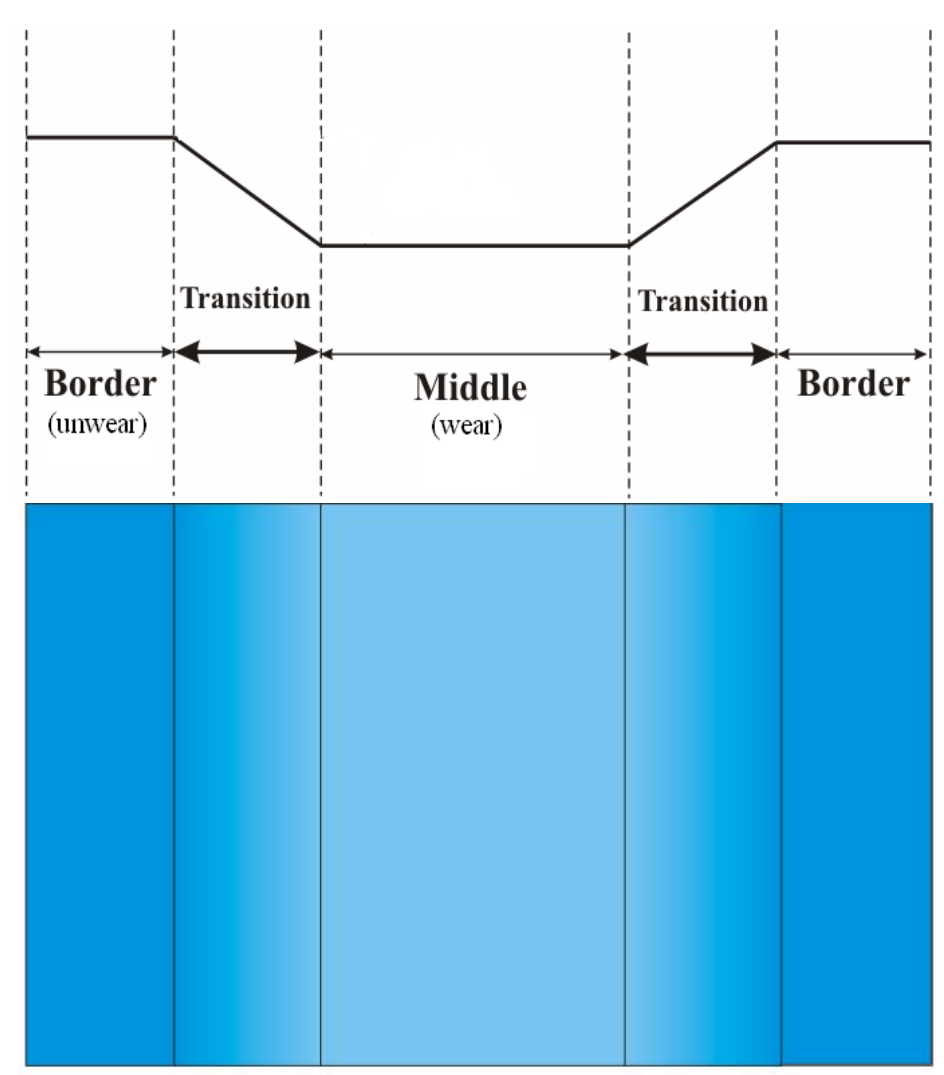

Theoretical representation 


\section{Features Validation}

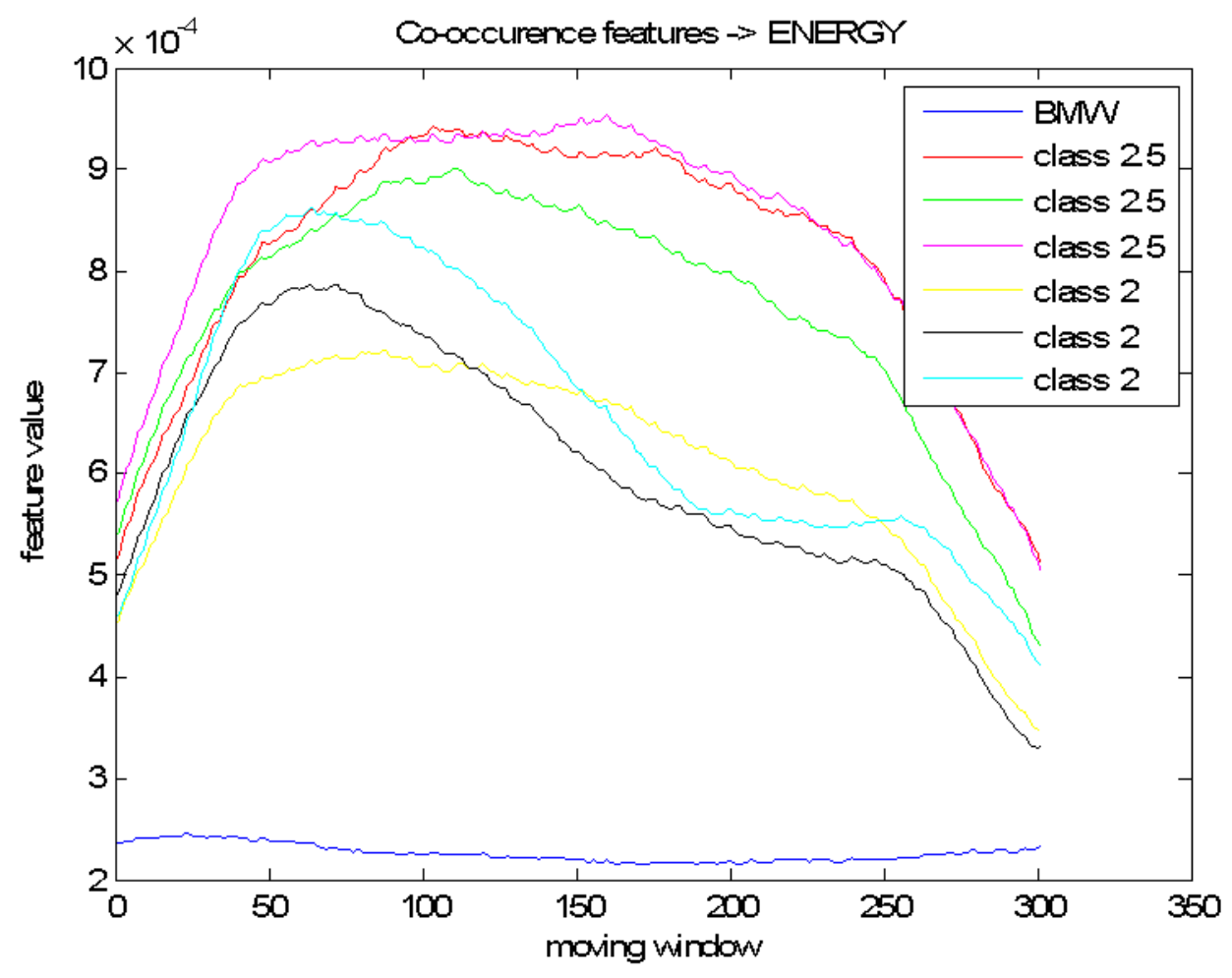

\begin{tabular}{|c|c|}
\hline Name of carpet & $\begin{array}{c}\text { Human expert } \\
\text { classification }\end{array}$ \\
\hline $20 \mathrm{kl} 517$ & BMW \\
\hline $20 \mathrm{kl} 517-2$ & 2.5 \\
\hline $20 \mathrm{kl} 517-4$ & 2.5 \\
\hline $20 \mathrm{kl} 517-6$ & 2.5 \\
\hline $20 \mathrm{kl} 517-8$ & 2 \\
\hline $20 \mathrm{kl} 517-10$ & 2 \\
\hline $20 \mathrm{kl} 517-12$ & 2 \\
\hline
\end{tabular}

Table of human expert

Haralick feature - Energy 


\section{What and why PCA?}

$>$ Principal Component Analysis is a useful technique used to reduce the dimensionality of large data sets, such as those from micro array analysis;

$>$ When there are more than three variables, it is more difficult to visualize graphically their relationships.

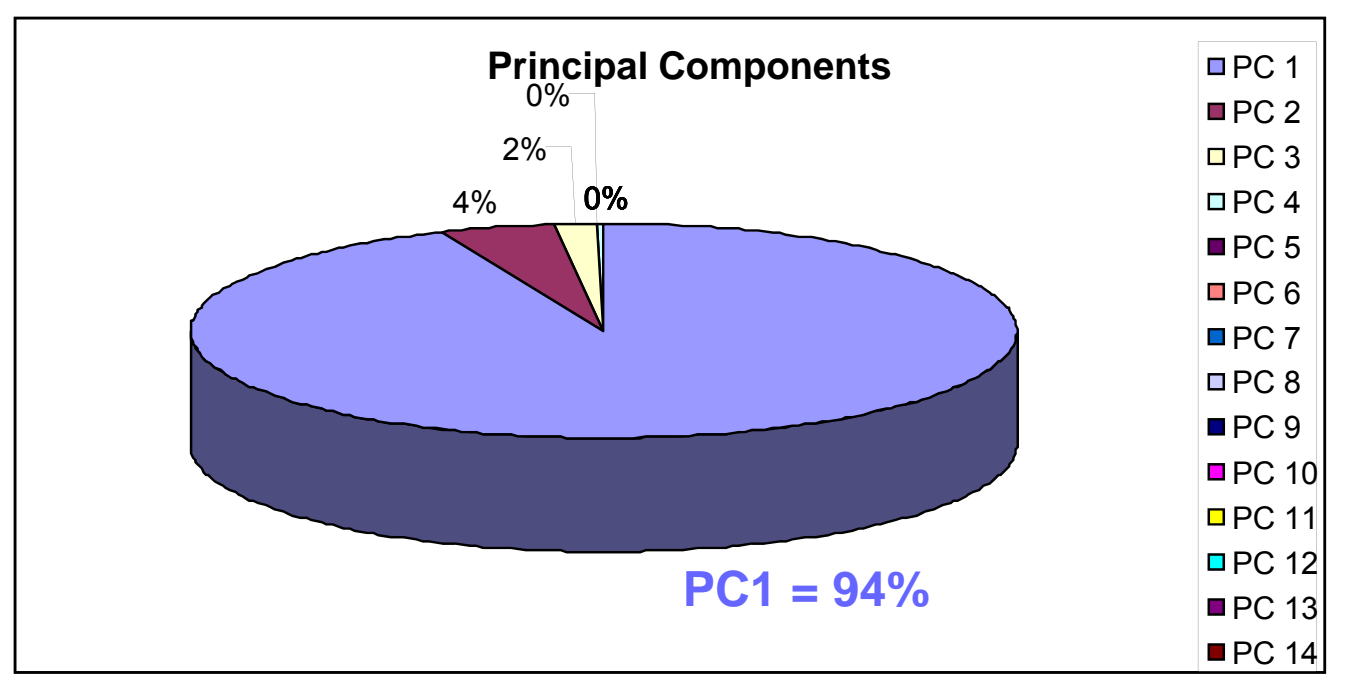




\section{PCA Results}

\section{PCA}

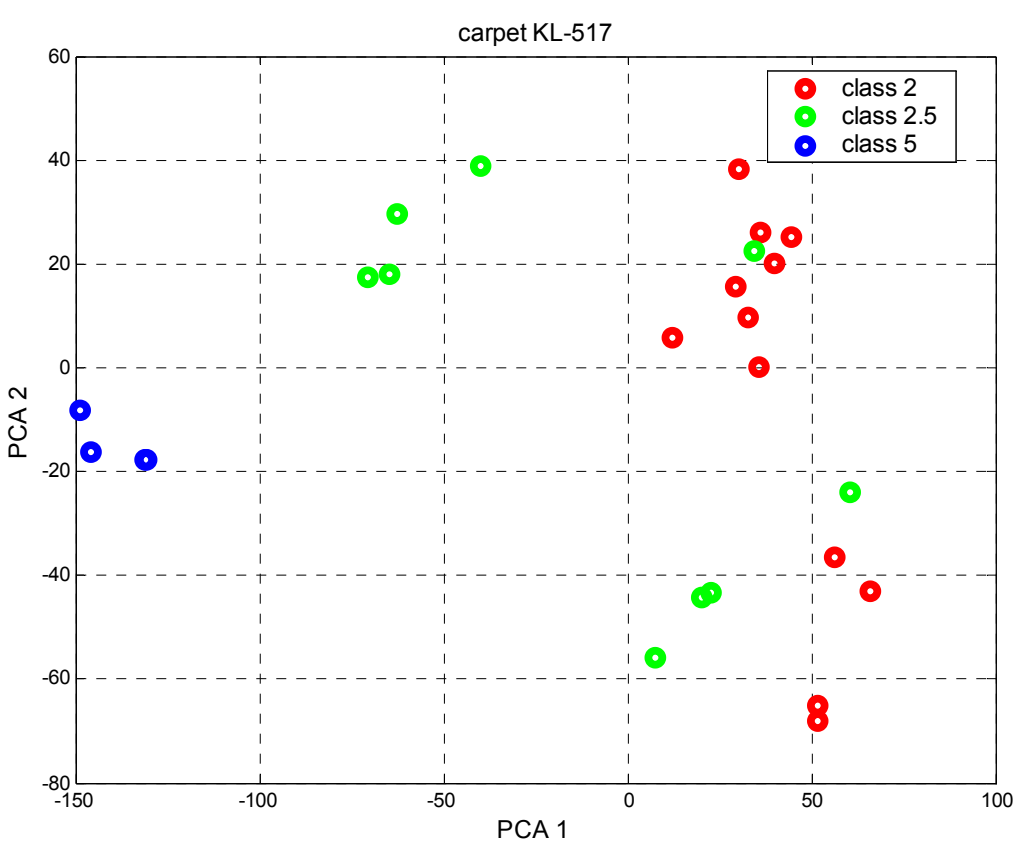

2D Plotting of first tow $P C$

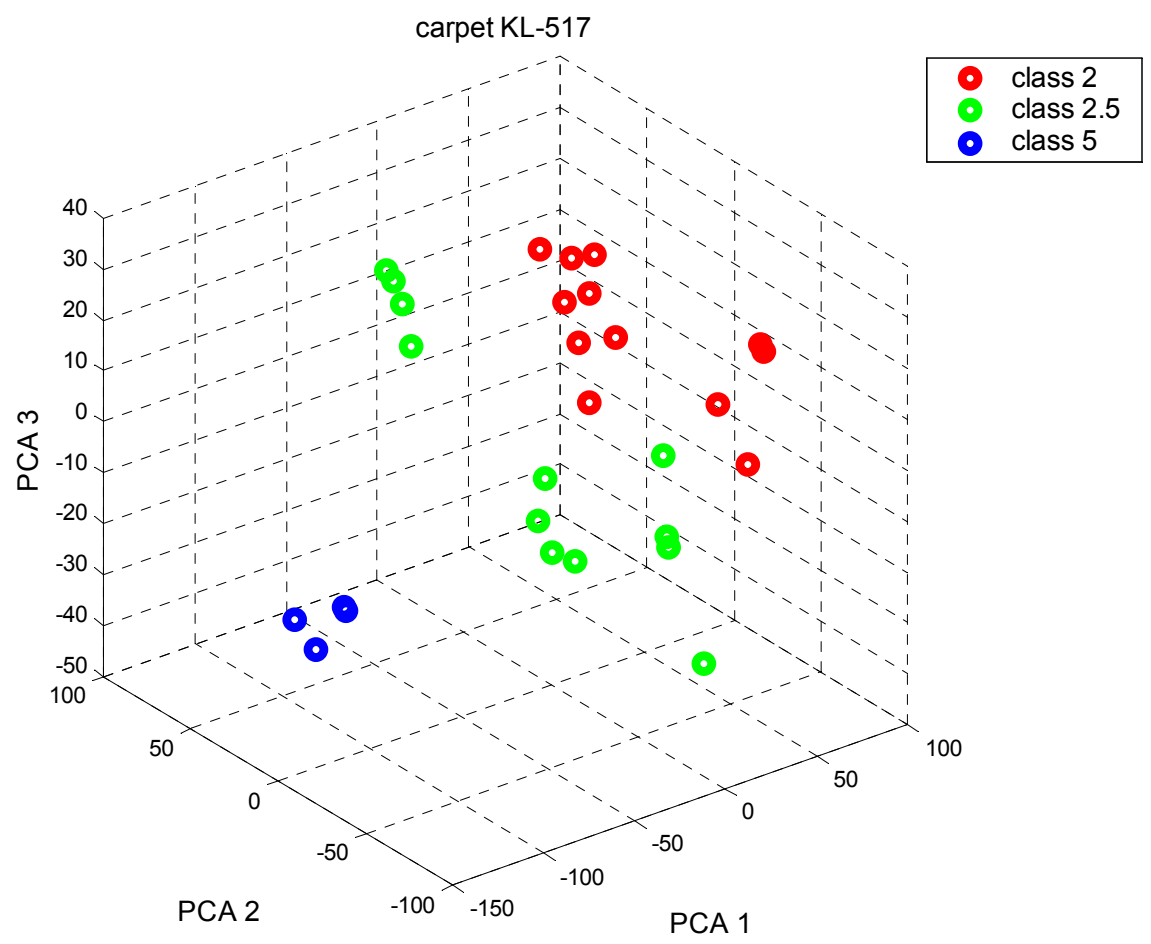

3D Plotting of first third PC 


\section{Features classification}

\section{Binary SVM}

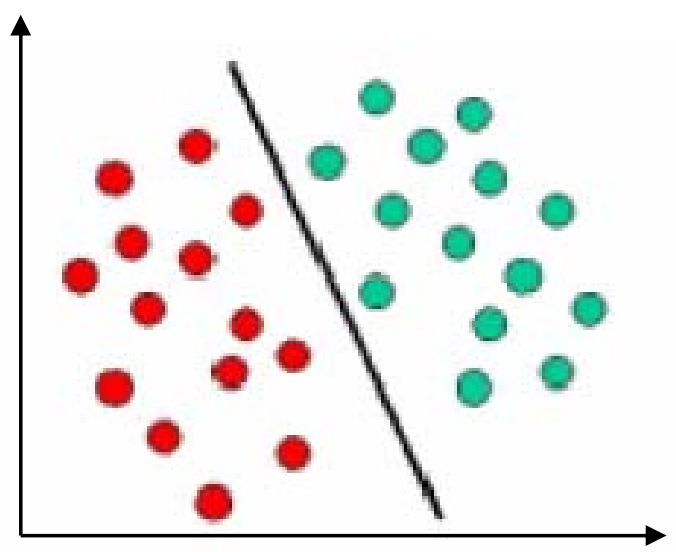

A separated hyperplane:

$$
w^{T} x+b=0
$$

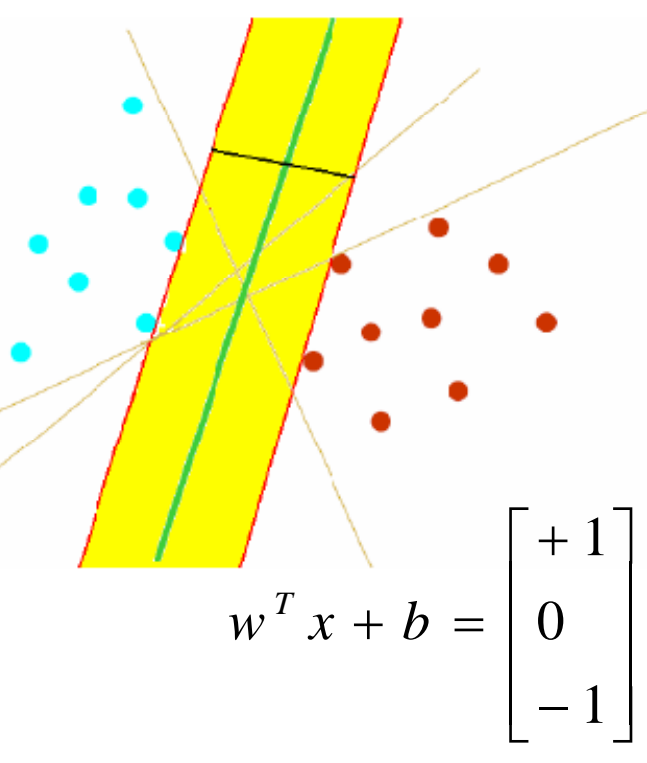

$$
\begin{array}{lll}
w^{T} x+b>0 & \text { if } & y=1 \\
w^{T} x+b<0 & \text { if } & y=-1
\end{array}
$$

Where $-\mathrm{x}$ is training data

$-y$ is indicator vector 


\section{Nonlinear - SVM}

\section{Features classification}

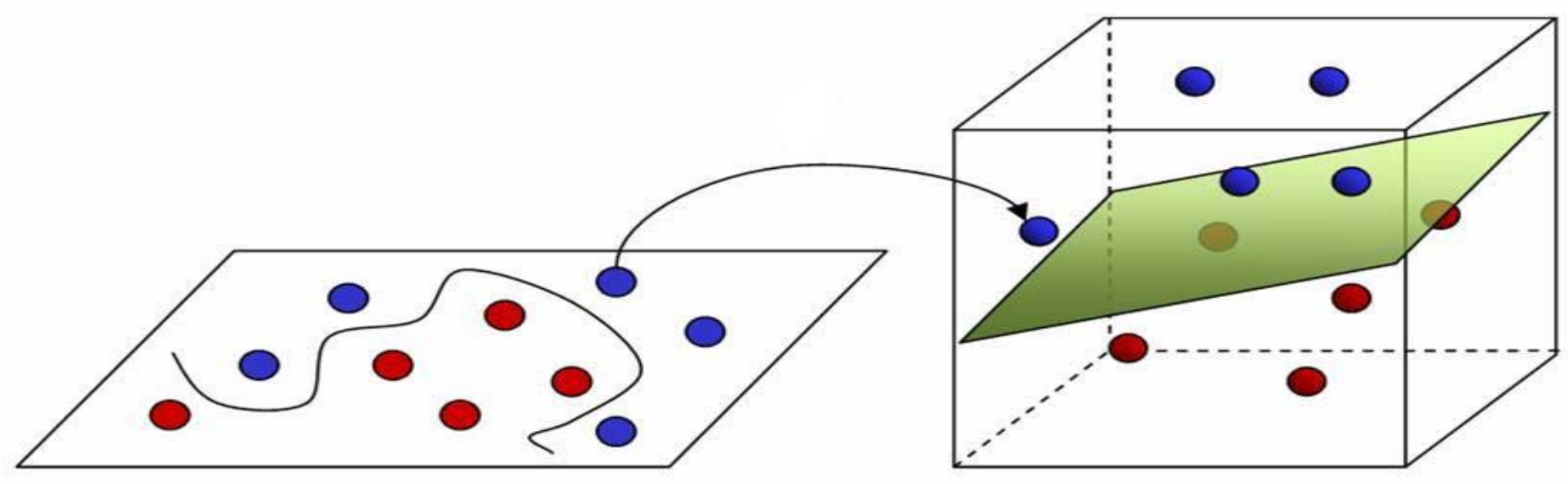

Input space

Feature space

$$
\begin{aligned}
& k\left(x_{i}, x_{j}\right)=\left(x_{i} x_{j}+1\right)^{p} \text { - polynomial kernel } \\
& k\left(x_{i}, x_{j}\right)=e^{-\|x-y\| / 2 \sigma^{2}} \quad \text { - Gaussian kernel }
\end{aligned}
$$

The separable plane will be:

$$
k\left(x_{i}, x_{j}\right) w^{T}+b=0
$$




\section{Multi - class SVM}

$>$ One - Against - All decomposition

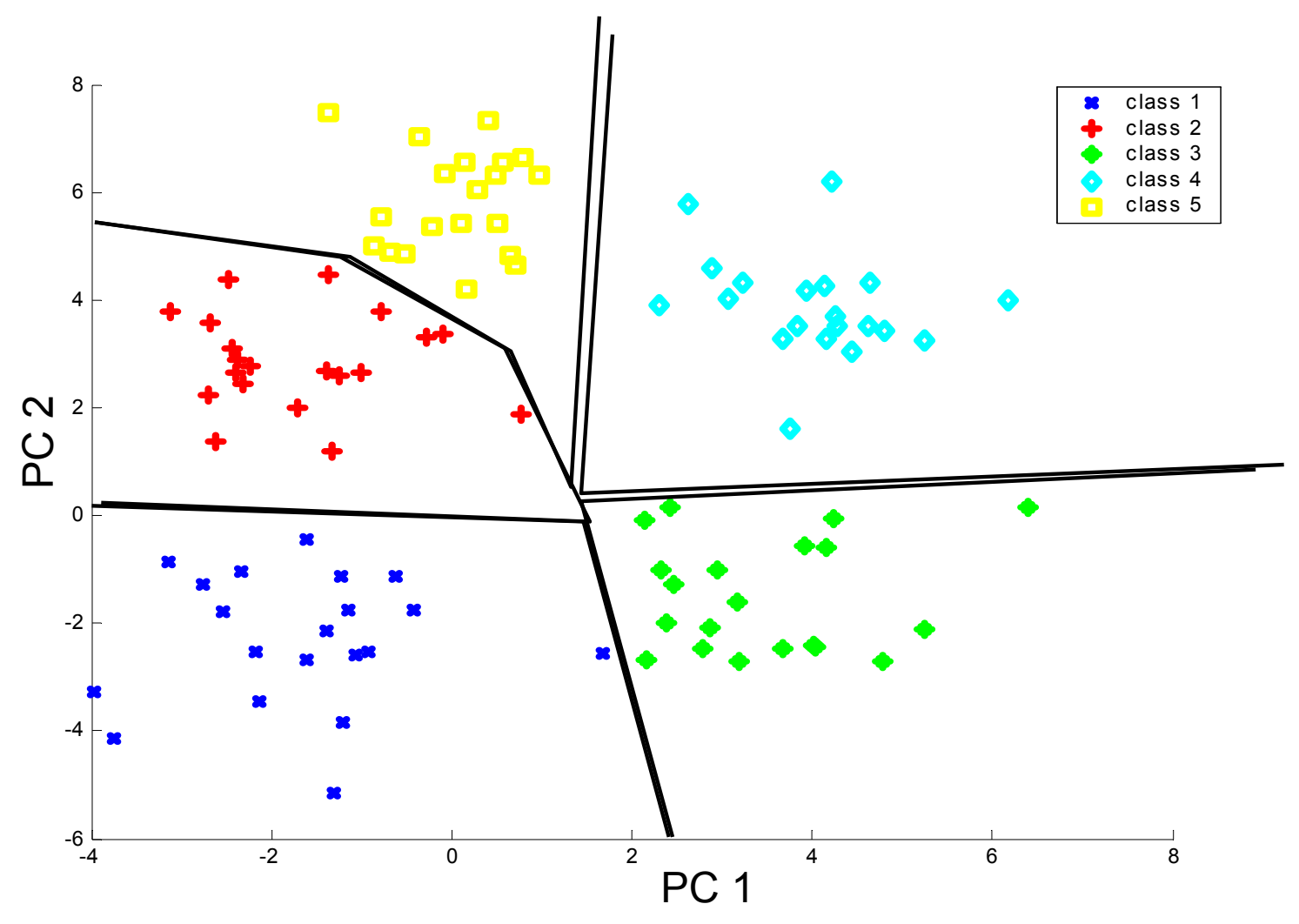




\section{Testing Results}

\section{Results}

$>$ For every carpet are used:

- 4 points for training

-1 point for testing

\begin{tabular}{|c|c|c|}
\hline Type of carpet & $\begin{array}{c}\text { Polynomial } \\
\text { kernel } \%\end{array}$ & $\begin{array}{c}\text { Gaussian kernel } \\
\%\end{array}$ \\
\hline A8 -501 & 84,4 & 84,4 \\
\hline A8 -701 & 84,4 & 100 \\
\hline Big 4 & 100 & 100 \\
\hline 20 KL 803 beige & 84,4 & 84,4 \\
\hline LA 7 & 84,4 & 100 \\
\hline Pr 84 & 100 & 100 \\
\hline 20 KL 517 & 100 & 100 \\
\hline Big 8 & 86 & 86 \\
\hline LA 9 & 72 & 100 \\
\hline 20 KL 803 & 100 & 100 \\
\hline Over all & 89,56 & 95,48 \\
\hline
\end{tabular}

Table of percentage classification 


\section{SVM Classification}

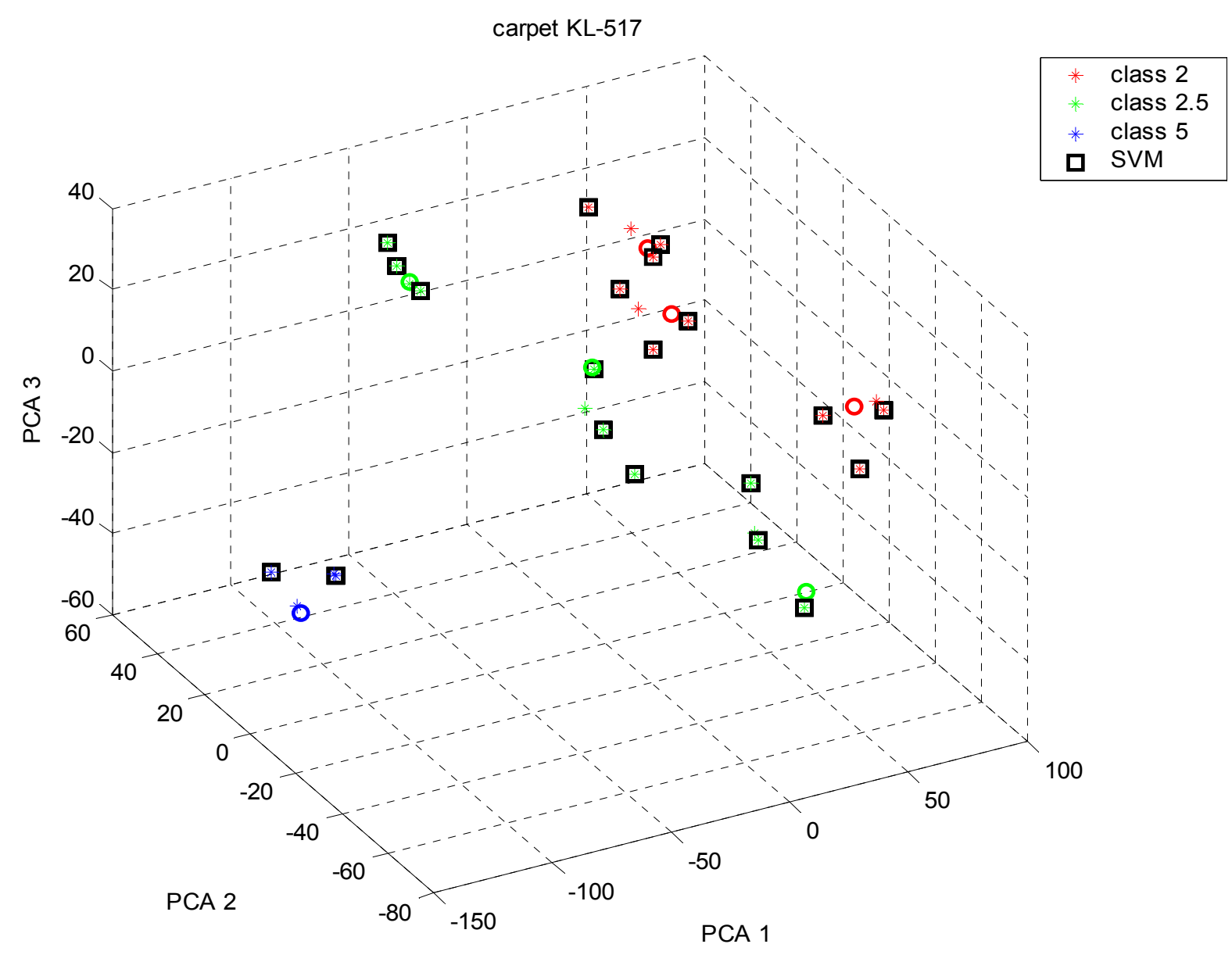

Classification for carpet KL-517 


\section{Conclusion}

$>$ Automated carpet classification can be achieved using co-occurrence matrix, PCA and SVM

> Polynomial kernel gives $88,32 \%$ over all classification

$>$ Gaussian kernel gives $94,24 \%$ over all classification 


\section{THANK YOU!}

